\title{
Once upon a time: from the early surveys to the epoch of the Northern Cross
}

\author{
Carla Fanti ${ }^{1}$ \\ Istituto di Radioastronomia \\ Via Gobetti 101 - 40129 Bologna, -Italy \\ E-mail: cfanti@ira.inaf.it \\ Roberto Fanti \\ Istituto di Radioastronomia \\ Via Gobetti 101 - 40129 Bologna, -Italy \\ E-mail: rfanti@ira.inaf.it
}

We present our personal view of the evolution, through the years, in the understanding the physics of the process, discovered in the 30s of the last century, that makes unknown celestial bodies strong emitters of radio waves. A variety of imaginative instruments (from the Sea Cliff to the Aperture Synthesis Interferometers) had to be invented to meet the performances required to face the new problems. The pioneering surveys carried out during those years, from the first "discrete source" (Cyg A) to the hundred- and thousand-source surveys of the mid-sixties, have played a fundamental role in finally understanding the physics of different classes of radio objects.

We shall further recall the impact that this discovery had to the lively, and sometimes heated, debate on cosmological theories. The concept that the Universe, as unveiled by the radio observations, is different from and complementary to the optically known Universe, has been a sort of cultural event and may have cleared the way to a greater open-mindedness in the exploration of the many facets of the Universe (X-ray, $\gamma$-ray cosmic radiations, new type of objects - quasars, pulsars and so forth)

To set this story up, we preferred not to use historical books, but to get a personal feeling of those year atmosphere. Hence we went through a lot of original scientific publications. It was really fascinating and sometimes intriguing and we ended up with some 150 articles (not all quoted in the present bibliography). What impressed us most was the prudence (sometimes, perhaps, reluctance) in accepting unpredicted discoveries or theories. In the light of the modern, sometimes hasty, attitude, early radio astronomers may look, nowadays, a bit uncertain or narrow-minded, but they probably just meant to be wise.

In order to squeeze everything into the allotted pages we had then to make choices, which are, obviously, personal and do not claim to be complete.

EXTRA-RADSUR2015 (*)

22-23 October 2015

Bologna, Italy

(*) This conference has been organized with the support of the Ministry of Foreign Affairs and International Cooperation, Directorate General for the Country Promotion (Bilateral Grant Agreement ZA14GR02 - Mapping the Universe on the Pathway to SKA)

\footnotetext{
${ }^{1}$ Speaker

(c) Copyright owned by the author(s) under the terms of the Creative Commons

Attribution-NonCommercial-NoDerivatives 4.0 International License (CC BY-NC-ND 4.0).
} 


\section{Introduction (1932-42 circa): It all started with Jansky.}

Everybody knows the story. In 1932 the physicist Karl Jansky serendipitously detects meterwavelength signals from the Milky Way (M.W.) which show a maximum in the direction of Sagittarius constellation (Jansky, 1933). He refers to it as cosmic statics (subsequently called cosmic noise and then Galactic radio emission). The news appears on the N.Y. Times $1^{\text {st }}$ page.

Other physicists and engineers, interested in this discovery, provide further information using very primitive instrumentation (e.g. Friis \& Feldman, 1937, Bell Labs; Potapenko \& Folland, 1936, CalTech). F. Zwicky, characteristically, urges that CalTech build a proper radio telescope. Millikan, (CalTech director) refuses to finance the project - 1000 US\$ (see Cohen, 1994).

Much work is done by Grote Reber (1937 and following years; see Reber, 1950 for an extended history of his own work). He scans the observable sky and detects signals at meter (but not at centimeter) wavelengths from a narrow strip centered on the galactic plane. The emission is quite smooth except for a few other peaks in addition to that in Sagittarius. Which mechanism could be the responsible for this radiation? It cannot be due to thermal free-free transitions (as supposed e.g. by Henyey \& Keenan, 1940) since there is no detection at high frequencies.

Moxon (1946) will prove that the 4- $\lambda$ spectrum of the M.W. emission in the range 40-200 MHz is non-thermal $\left(S \propto \lambda^{0.7}\right)$. Townes (1947) will demonstrate that the M.W. luminosity at decameter wavelengths requires $T_{b} \approx 10^{5} \mathrm{~K}$, too high to be thermal.

\section{The first discrete radio sources: from one to a-few source surveys (1946-49)}

During the II world war (February 1942) two German warships could escape, undiscovered, through the Channel: all British radars are jammed by strong radio noise of unknown origin, for sure not due to enemy disturbing radars. J.S. Hey, a physicist enrolled by the British army as a radar controller, discovers that these disturbances come from the Sun (active at that time). A shocking result! Obviously this discovery will be kept top-secret until the end of the war!

After the war, many people, including Hey (1946a), study the radio-Sun and find that its emission varies in amplitude from day to day (but also with $<1 \mathrm{~min}$ periods). The variable part of the emission shows circular polarization. (We omit Sun studies because not relevant here).

If the Sun (a star...) can emit radio waves, could the integrated emission of all Sun-like stars in the Galaxy, individually unresolved, explain the observed M.W. smooth radio emission (as for the optical M.W. before Galileo succeeded in resolving it with a telescope)? Greenstein et al. (1946) prove that the expected emission would be $10^{-12}-10^{-9}$ times the observed one.

Using an abandoned war radar, properly modified, Hey et al. (1946b) map the M.W. at 64 $\mathrm{MHz}$ with a $30^{\circ} \times 12^{\circ}$ beam. In this process they come across a region, $<2^{\circ}$ in size, in the Cygnus constellation, showing intensity variations of $\approx 15 \%$ with a $<1 \mathrm{~min}$ periods, as in the Sun.

Using the Sea Cliff interferometer in Australia, Bolton \& Stanley (1948) confirm Hey's discovery and estimate the size of the varying region to be $<8$ '.

Is the emission mechanism in the M.W. the same of the Sun?

A new concept is introduced: a discrete source of radio emission, distinct from the M.W. diffuse emission is present in the Cygnus region (hereafter named Cyg A).

Given its estimated angular size, $C y g A$ brightness temperature results very high $\left(T_{b}>410^{6} \mathrm{~K}\right)$.

Cyg $A$ is not a unique object: using the Sidney Sea interferometer, Bolton (1948) discovers six more discrete sources, less than 15'-60' in size. Four are in the Taurus, Coma Berenices (later corrected to Virgo), Hercules and Centaurus constellations. Bolton suggests that such sources be the brightest members of an unknown class of anomalous stars, out of the main sequence, whose integrated emission might produce the diffuse non-thermal galactic radio emission, in addition to the thermal emission from interstellar gas and to that from normal (Sun-type) stars.

While searching for polarization in Cyg $A$ with a Michelson-type radio interferometer at $80 \mathrm{MHz}$, Ryle \& Graham-Smith (1948), discover two more discrete sources: one in Ursa Major, the other, stronger than Cyg A, in the constellations of Cassiopeia. They confirm the intensity variations in $C y g A$, some very rapid ( $\approx 20 \mathrm{sec}$ ?), assumed intrinsic to the source (but see below). 
At $1 \mathrm{pc}$ distance such variations imply a linear size of the order of the Sun size. $\boldsymbol{C y g} \boldsymbol{A}$ must be a (radio-)star, with $T_{b}>10^{14} \mathrm{~K}$. Finally they find that neither Cyg A nor Cass A are circularly polarized, contrary to the Sun, so that these radio star are not Sun-type. (Stanley \& Slee, 1950 and Ryle \& Hewish, 1950 will prove the ionospheric origin of radio stars variability).

Meanwhile Bolton et al. (1949) using the New Zeeland Sea interferometer provide "accurate" (5'-6') positions for Tau A, Virgo A and Cen A. By comparing radio with catalogue optical positions, they suggest possible counterparts (Bolton \& Stanley, 1949): Taurus A is close to the SNR Crab Nebula, the two others within 8' of the nebulae NGC 4486 and NGC 5128, classified, in the Shapley \& Ames catalogue as extragalactic. The authors, however, comment that "Neither of these two objects has been resolved into stars, so there is little definitive evidence to decide whether they are true extragalactic nebulae or diffuse nebulosities within our Galaxy". Bizarre comment since Hubble \& Humason (1931) had classified NGC 4486 as an E0 in Virgo cluster and measured a recession velocity of $800 \mathrm{~km} / \mathrm{sec}$, equal to the cluster systemic velocity.

In any case none of the considered discrete radio source is associated with a stellar object.

By the end of the 40s, a handful of radio stars have been discovered, on top of the diffuse radio emission of the M.W., but there is no way yet to find whether a link exists between the two processes. Then, for some years, the study of the nature of radio stars will remain strictly bound to that of the origin of the galactic radio emission. We briefly discuss this in the next section.

\section{Non-thermal radio emission of the Milky Way: two scenarios (1950- 60)}

1) Individually unresolved stars.

Ryle (1950) suggests that a sufficiently high number of Strongly Magnetized fast rotating stars (discussed by Alfvén, 1937) may be responsible for the M.W. non-thermal radio luminosity. A magnetic field of $\approx 5000 \mathrm{G}$ and a peripheral rotational velocity of the order of $10^{7} \mathrm{~cm} / \mathrm{sec}$ may create electric fields of such an intensity that electrons can be accelerated at very high energies. These electrons may produce luminosities $>10^{4}$ times that of the Sun. Similar star models are being considered in these years also by Unsöld (1949) and Alfvén \& Herlofson (1950).

- Westerhout \& Oort (1951) assuming that radio stars are spatially distributed like the most common optical stars ( $\mathrm{G}$ and $\mathrm{K}$ dwarf) compare the expected spatial distribution of the radio luminosity with that observed at $100 \mathrm{MHz}$ by Bolton \& Westfold (1951). The result is unsatisfactory at high galactic latitudes and in the anticenter. The suggestion is that an isotropic, possibly extragalactic, contribution may be present in addition to the galactic one.

- In 1953 Baade \& Minkowsky, thanks to their Optical Identifications (Sect. 5), reluctantly, will weaken the radio star hypothesis. The discrete source counts by several authors (1955, Sect. 4) will transform most radio stars into extragalactic radio sources.

\section{2) Magnetobremsstrahlung (Synchrotron - Oort \& Walraven, 1956)}

K.O. Kiepenheur (1950) shows that the intensity of the galactic radio emission can be explained by relativistic electrons (expected to be present in Cosmic Rays, and discovered later by Earl, 1961) accelerated in the galactic magnetic field (recently discovered by W. Hiltner, 1949 and by J.S. Hall, 1949 from the starlight polarization by dust grains). Kiepenheur calculates that $\approx 1 \%$ of $e^{-}$in Cosmic Rays of $10^{8} \mathrm{eV}$ and $H_{\text {gal }} \approx 10^{-6} \mathrm{G}$, in a layer $300 \mathrm{pc}$ thick, could reproduce the observed M.W. luminosity at $100 \mathrm{MHz}$. This simple process does not imply unusual stars to explain the Galactic emission and is taken into serious consideration by theoreticians in the Soviet Union (Ginzburg, 1953, Shklovsky, 1953 and others). They further develop the theory and find it encouraging also for discrete radio sources. They also stress that the signal is expected to be linearly polarized and polarization measurements (radio \& optical) are suggested by Shklovsky as an important test. By the beginning of 1953 the radio star hypothesis is definitively abandoned in USSR.

In the Western countries the synchrotron model for M.W. will be accepted only by the late $50 \mathrm{~s}$. -Mills (1959a) will successfully decompose the M.W. radio spectrum between $3.5 \mathrm{~m}$ (Sydney Cross, Sect. 6) and $22 \mathrm{~cm}$ (by Westerhout, 1958; Dwingeloo, 25-m dish) assuming that the 
conspicuous non-thermal fraction of the Galaxy radio radiation is due to synchrotron. Notable his comments (Mills 1959b,a): "The assumption in the early days of radio astronomy that the galactic emission resulted from the integrated output of large numbers of radio sources, has now been largely superseded by the assumption that it originates in a diffuse medium comprising relativistic electrons moving in magnetic fields ...... Although the synchrotron emission has not been established as the only non-thermal process, no other has been suggested that can be seriously considered.". To make synchrotron model conclusive, radio polarization would have been required, but this will come later on by Westerhout et al. (1962) (but see also positive indications by Thomson, 1957 and Razin, 1958). At this late epoch the origin of the diffuse galactic non-thermal radio emission begins to be disentangled from that of the discrete sources.

About discrete sources:

- Oort \& Walraven (1956) will detect optical polarization in the Crab nebula diffuse emission, confirming earlier results by the Soviet astronomers Vashakidze \& Dombrovsky (1954). The polarization at radio wavelengths will come by Mayer at al. (1957).

- Mayer et al.(1962) will detect radio polarization in Cen A and Cyg A (plus a few other sources).

- Burbidge (1956) will apply synchrotron (that he calls "acceleration radiation") theory to the study of the optical jet of NGC4486/M87 (Virgo A) whose optical polarization had just been discovered by Baade (1956). Burbidge (1959) will then apply the theory to a dozen of extragalactic plus a few galactic radio sources and will show how to compute source minimum (equipartition) energy and magnetic field, thus quantifying what will be known as the "energetic problem".

But let's go back to our chronological tale.

\section{Few-tens source surveys: Impact on the nature of "radio stars" (1950-55)}

By the end of the 40s (Sect. 2) a small number of radio stars are known and it begins to be evident that they represent only the "tip of the iceberg".

High resolution more powerful flexible instruments are quickly built by several independent groups to extensively survey the sky. In these years lists counting up to 100 radio stars are compiled (see Table). The observing frequencies are $\lesssim 200 \mathrm{MHz}$ and the limiting flux densities of the order of tens of Jy. The angular resolution is of several degrees for single dishes and fringe spacing $\approx 1^{\circ}$ for interferometers. Positional accuracy is of a few arcmin. Each of these surveys covers a wide region of the visible sky. They have large reciprocal overlaps and often show discrepancies in the measured parameters. In total some 150 independent discrete radio sources are now available for very useful, although statistically still limited, studies.

\begin{tabular}{|c|c|c|c|c|c|c|}
\hline Authors & $\mathrm{N}$ & $\begin{array}{c}v \\
(\mathrm{MHz})\end{array}$ & $\begin{array}{c}S_{\text {lim }} \\
(\mathrm{Jy})\end{array}$ & $\begin{array}{l}\text { Accuracy/ } \\
\text { Prim.beam }\end{array}$ & $\begin{array}{l}\text { dec. sky } \\
\text { coverage }\end{array}$ & Instrument \\
\hline $\begin{array}{l}\text { Stanley \& Slee } \\
(1950)\end{array}$ & 22 & 100 & 100 & $9^{\circ} \times 17^{\circ}$ & $-50^{\circ} \div+50^{\circ}$ & Sea interferometer \\
\hline $\begin{array}{l}\text { Ryle, Smith \& } \\
\text { Elsmore(1950) }\end{array}$ & 50 & 81 & 30 & $\begin{array}{l}\approx 1^{\prime} \times 5^{\prime} \\
1.5^{\circ} \times 90^{\circ}\end{array}$ & $+12^{\circ} \div+82^{\circ}$ & Phase switch Interferometer \\
\hline Mills (1952a) & 77 & 101 & 50 & $\begin{array}{l}2 \mathrm{~min}(\mathrm{RA}) \\
14^{\circ} \times 24^{\circ}\end{array}$ & $-90^{\circ} \div+50^{\circ}$ & $\begin{array}{l}\text { Michelson-type Interferometer } \\
2 \text { EW baselines }\end{array}$ \\
\hline $\begin{array}{l}\text { H-Brown\& Hazard } \\
\text { (1953) }\end{array}$ & 23 & 158 & 3 & $2^{\circ} \times 2^{\circ}$ & $+38^{\circ} \div+68^{\circ}$ & JB $218 \mathrm{ft}$ transit Paraboloid \\
\hline $\begin{array}{l}\text { Shain \& Higgins } \\
\text { (1954) }\end{array}$ & 37 & 18.3 & 2500 & $17^{\circ} \times 17^{\circ}$ & $-52^{\circ} \div-12^{\circ}$ & Sydney $30 \lambda / 2$ Dipole array \\
\hline $\begin{array}{l}\text { Bolton, Stanley \& } \\
\text { Slee (1954) }\end{array}$ & 104 & 100 & 50 & $\begin{array}{l}\text { Fringe } \\
\text { Spacing } 1^{\circ}\end{array}$ & $-50^{\circ} \div+50^{\circ}$ & Sea interferometer \\
\hline
\end{tabular}

- Radio source spatial distribution in the Galaxy and number-flux density relation (Log N-Log S)

Mills (1952a) describes a bimodal spatial distribution: the strong radio sources ( $S \geq 300 \mathrm{Jy}$ ) are mostly concentrated within $|b|<12^{\circ}$. These are referred to as Class I, and suggested to be Galactic (in the majority of cases: e.g. $C y g A$, at $b=5.8^{\circ}$ will be recognized as extragalactic). The much more numerous weak radio sources have a uniform distribution in $|b|$ (Class II). They are 
suggested to be either intrinsically weak and very close-by, or very strong and extragalactic. Note that Ryle et al. (1950) don't find this dichotomy, but this could be due to the fact that Class I sources will often found to be extended and therefore likely be missed by the interferometric observations.

About source counts, defined as $N(>S) \propto S^{-\delta}$ (called "ogive" at that epoch) Mills finds $\delta=0.75$ for Class I, consistent with a random disk distribution (similar to that of mag $<15$ stars). The galactic nature is further supported. For Class II, $\delta=1.5$, typical of a uniform space distribution of either feeble galactic emitters at a distance sufficiently short for their distribution to be unaffected by the M.W. finite dimensions, or of very strong extragalactic objects.

Mills' results are confirmed by Bolton et al. (1954) except that their $\log N$-Log $S$ for Class II sources steepens up to $\delta \approx 2$ for $S \lesssim 100 \mathrm{Jy}$. They don't make any comment, but this is the same result that Ryle will find with the source counts of from the $2 C$ survey (see Sect 6).

Mills (1952a) presents a complete and open-minded analysis of the situation, which seems to prelude to the extragalactic hypothesis, but in the abstract of the paper he states: "Although some of the evidence is suggestive, it is insufficient for any positive conclusion to be formed". The idea that a conspicuous population may exist of extragalactic radio sources is hazardous, hard to digest!

Interesting the comment in Ryle et al. (1950): "it does not seem possible to suppose that the majority of the observed radio stars are outside our Galaxy because of theoretical difficulties encountered in attempting to explain the very great intensities of the more intense radio stars......"

Since no correlation seems to exist between radio positions and known (from catalogues) optical positions for Class II sources, en passant Mills (1952a) says that "the possibility that a rare type of galaxy is involved is not ruled out and is, in fact, slightly strengthened by a tendency for positions of the stronger sources to occur in the direction of cluster of galaxies"

H. Brown \& Hazard (1953) support the galactic nature of Class I radio sources and, having measured a diameter of a few arcmin for Cass A, comment that "If this source is typical of Class I, this Class cannot be identified with bodies of stellar dimensions". Also Mills (1952a), from sparse information in the literature, concludes that "the evidence does seem to suggest that the class I sources could be nebulous objects of considerable size".

- Radio spectra $\left[S(v) \propto v^{-\alpha}\right]$

Very little is known so far. At this epoch Stanley \& Slee (1950) determine 5-v (40, 60, 85, 100, $160 \mathrm{MHz}$ ) spectra for the four strongest sources in their list (Cen A, Cyg A, Virgo A, Tau A). For the first three the spectrum is consistent with a power law with $\alpha=1$, while $\alpha=0$ for Tau $A$ (Crab Nebula). The latter slope is compatible with a thermal transparent spectrum (but see Oort \&Walraven, 1956, Sect. 3, who will prove that this is synchrotron emission).

More radio spectra will come by the end of 50s - beginning of 60s (Sect. 7).

- Tentative Radio-Optical associations.

By the end of the 40s the only suggested optical counterparts for radio sources are those by Bolton et al.(1949), Bolton \& Stanley(1949). A few more tentative candidates are now added.

- Ryle et al. (1950) indicate M 31, M 33, M 51, M 81 \& M 101 (all Spirals) for five radio stars.

- Mills (1952b) measures positions with arcmin accuracy for Cyg A, Cen A, Virgo A, Tau A,

Fornax $A, \&$ Hydra $A$. He confirms the $I D$ by Bolton for the first four objects while finds no notable optical object for Hydra A and Fornax A.

- H.Brown \& Hazard (1953) suggest the association of a radio source with SNR 1572 (Tycho). Four Class II sources are suggested to be associated with Spiral (M31\& M51, already suggested by Ryle et al.,1950, plus M 891\& NGC 4258). They note that the radio/optical luminosity ratio for late type Spirals is roughly constant and similar to that of the M.W. and Andromeda. Another source is associated with NGC 1275, a peculiar galaxy in Perseus cluster. Considering also Virgo A (M87) and Cen A (NGC 5128), they mention the possibility that a type of galaxies exists in which the radio luminosity is much larger than the optical one, "thus the poor correlation of the sources with the bright extra-galactic nebulae cannot now be taken to rule out their extragalactic nature, for the visible object may be exceedingly faint". Do radio studies probe farther than optical? 


\section{Confirmed Optical Identifications (ID) (1953-54)}

In the first 50s about 15 radio sources are tentatively associated to visible objects. A proper observing program is then started by Baade \& Minkowski to verify the suggested IDs. By cross checking the multiple independent positions of individual radio sources they manage to have radio positions accurate enough $\left(\approx 2^{\prime}\right)$ to afford pointed observations with the Palomar $48^{\prime \prime}$ and 200 " telescopes. This accuracy is sufficient since the observed radio sources are the strongest and some 0ptical objects are quite prominent. Minkowski reports on preliminary results at the J.B.Symp (H.Brown, 1950). The complete work is published in Baade \& Minkowski (1954a,b).

Thanks to improved positions by G. Smith, they identify with certainty Cass A, Puppis A and $C y g A$. The first two will be understood, later on, as SNRs.

The most shocking revolutionary results are from $C y g A$ whose counterpart is a 16 mag. object, at the center of a cluster, with redshift $z=0.056$ (quite high for the epoch) and hence at a distance of $31 \mathrm{Mpc}\left(H_{0}=540 \mathrm{~km} / \mathrm{sec} / \mathrm{Mpc}\right.$ at this time). The corresponding radio luminosity is enormous: Cyg A could be detected in radio even at a 10 times larger distance, out of reach of the 200" telescope, i.e. when optically invisible. This result likely explains (in addition to the poor radio positions) why so few optical counterparts have been found so far. The optical object resembles two galaxies in collision (hence the first theory to explain the enormous energies involved). Today we know that this appearance is due to dust lanes.

In a footnote, they fairly acknowledge: "this coincidence was already noted in 1951 by Mills and Thomas .... but it seemed unlike at that time that a distant galaxy could be the radio emitter".

The ID of Virgo A, Cen A and Perseus A are also confirmed. All three are Elliptical galaxies with some peculiarity: the first one has an optical jet, the two others a dust lane which is interpreted as a dusty Spiral colliding with an Elliptical galaxy. In addition they confirm eight optical $I D$ with $S b$ and $S c$ galaxies, earlier indicated mostly by Ryle et al. (1950) and H.Brown \& Hazard (1953). The authors note that "no ID is with stars" (although, admittedly, they would be difficult to perform without an arcsec radio position accuracy, comparable to the optical one).

At the J.B.Symp Ryle comments: "It appears that we have both galactic and extragalactic sources and the question arises as to whether the galactic sources can account for the radiation from the Galaxy"

And even more Baade \& Minkowsky (1954a) note that "Under the impression that all sources identified so far are extended or of extragalactic origin, the concept of stars as radio sources has dropped into the background. It seems necessary to emphasize that an interpretation of the distribution of the general galactic radiation meets great difficulties without the assumption that radio stars exist"

Does radio star hypothesis begin to creak? Synchrotron model is still ignored for the M.W. non-thermal diffuse radio radiation. It takes a long while to break down prejudices!.....

\section{1000s of source surveys: Radio astronomy jumps into cosmology (1955-60)}

Radio astronomers have technologically grown up and are able to build very large sensitive instruments which produce extended surveys. Basically the two groups of Ryle and Mills dominate the scene:

- Shakeshaft et al (1955) $2 C$ survey - 4-element interferometer, $v=80 \mathrm{MHz}$; primary beam $1^{\circ} \times 7.5^{\circ}$, positional accuracy $2^{\prime} \times 12^{\prime}$ for strong sources; $S_{\text {lim }}$ a few Jy; $-38^{\circ}<$ dec $<83^{\circ}$;

1936 radio sources

- Mills et al. (1958) [see Mills et al.(1961)]: $\boldsymbol{M S H}$ survey - Sidney Cross (450 $\mathrm{m} \times 450 \mathrm{~m}) ; \mathrm{v}=85$ $\mathrm{MHz} ; \mathrm{HPBW}=50^{\prime} ; S_{\text {lim }} \approx 8 \mathrm{Jy} ;-80^{\circ}<d e c<+10^{\circ}$ (in 3 parts); 2270 radio sources

What happens then is probably known to everybody and we recall it concisely here. For an extended report see contributions by Scheuer and by Sullivan III in Bertotti et al. (1991).

From the $2 C$ catalogue a $\log N-\log S, N(>S) \propto S^{-\delta}$, with large statistics and ample flux density range, is quickly obtained. The result is really upsetting: the ogive slope, $\delta=1.5$ at high flux densities, sharply steepens below $\approx 80 \mathrm{Jy}$, reaching $\delta=3$. 
Ryle (1955) presents these results at the Halley Lecture in Oxford and discusses whether it is possible to model a proper distribution of radio stars in the Galaxy which reproduces both the shape of the $\log N-\log S$ and the isotropic distribution of the radio sources. The answer is "no" and Ryle has to conclude: “... we must abandon the idea of explaining the Log $N$-Log $S$ curve in terms of sources within the Galaxy and consider the alternative hypothesis, that the main class of radio stars is external to the Galaxy”. Scheuer (in Bertotti et al.,1991) comments: “... it must have been in the winter of 1953-54 that M. Ryle's attitude on radio stars changed almost overnight". At this point Ryle considers the cosmological implications of his results in terms of the Steady State Theory (SST) (Bondi \& Gold, 1948 Hoyle, 1948) and of evolutionary theories. Since in the SST the slope of the $\log N$-Log $S$ must be $\leq 1.5$, he concludes that “... if most of the radio stars are external to the galaxy, and this conclusion seems hard to avoid, then there is no way in which the observations can be explained in terms of Steady State Theory".

The Universe appears to be evolving! Cosmologists have strong negative reactions: the great cosmological debate has commenced ... - Pawsey (1957), at Jodrell Bank IAUS n.4 on Radio Astronomy (1955), presents a preliminary $\log N-\log S$ from the first section of the MSH survey (MS survey, 383 radio sources, Mills \& Slee, 1957). The slope (for class II) is 1.65 , compatible with $\delta=1.5$, much lower than Ryle's.

- Bolton (1956), after IAUS n. 4, in a letter to "Observatory" shows that the strong steepening of the $2 C \log N-\log S$ is due to confusion effects, namely there are too many weak sources in the primary beam (he mentions here his own personal experience; Sect. 4). Ryle and colleagues are actually aware of the confusion problems, but they had (wrongly) thought that the confusion would make the faint sources disappear (in Bertotti et al., 1991) thus flattening the source counts.

Ryle and Mills exchange some correspondence, but there has never been a real collaboration to solve the discrepancy in $\log N-\log S$.

Mills \& Slee (1957) compare their own preliminary $M S$ catalogue with the $2 C$ in the common area and states that "the two catalogues are almost completely discordant".

At the famous Paris IAUS n.9 (1958) the heated debate on SST continues, not only between Ryle and Mills but also with cosmologists. In the Symposium concluding lecture, Minkowski, quoting Hoyle and McVittie, comments that even reliable source counts may not be adequate to select a unique cosmological model. But this is not the point at present: the question is not how to select a unique cosmological model, rather whether or not the Universe is evolving! ....

The debate on source counts and on their interpretation continues.

Edge et al. (1958) very quickly move the frequency of the $2 C$ interferometer to $v=159 \mathrm{MHz}$, obtaining a 4 times smaller primary beam, hence less confusion. The limiting flux density, $S_{\text {lim }} \approx 10 \mathrm{Jy}$, is $2-3$ time higher than that of the $2 C$; the sky coverage, $-22^{\circ}<d e c<+71^{\circ}$, is comparable. They soon produce the $3 C$ survey, which counts 471 radio sources, and admit that

"One quarter of those in the previous survey at $81.5 \mathrm{Mc} / \mathrm{s}$ are confirmed".

The new slope of the $\log N-\log S$ is $\delta=2.2$, lower than the previous one, but still higher than Mills'. After three years of refinements, confusion corrections (Scheuer, 1957), re-observations with the new Aperture Synthesis Radio Telescope at $178 \mathrm{MHz}$ (Sect.8), the first revision of the $3 C$ survey (3CR) is ready (Bennett, 1962): it consists in a "complete" sample of 328 strong (>9 Jy) radio sources at $d e c>-5^{\circ}$. Slowly $\delta$ will converge to $1.8 \pm 0.12$, close to Mills' (Scott \& Ryle, 1961; Bennett, 1962, ..... until Gower, 1966 with the 4 C survey).

The two contending groups still disagree about the compatibility (Mills) with Steady State Theory or not (Ryle). Scheuer (in Bertotti et al., 1991), referring to his own visit at CSIRO says: "..... I got to know him (Mills) a little and we got along pretty well, though we never did agree about radio sources". Equally sharp remains the diatribe between those in favor and against the SST.

In 1965 the discovery of the Microwave Background Radiation closes the debate on SST. Not for everybody, however..... Scheuer (in Bertotti et al, 1991) comments: "After all, nearly all astronomers agreed that the Universe must be evolving .... In a sense, the debate continued with many of the same participants on grounds that had shifted again; this time it was about the redshift of quasars, and hence whether quasars could tell anything about cosmology" 
7. Surveys at high frequency \& resolution: the physics of radio sources (early 60s)

- High frequencies and radio spectra $\left(S \propto v^{-\alpha}\right)$

So far, surveys have been performed at meter wavelengths only while, from the Synchrotron theory (now accepted, Sect. 3) higher frequencies can contribute useful information. At the end of the 50s Whitfield (1957) compiles multi-v (up to $\sim 600 \mathrm{MHz}$ ) spectra of $\sim 30$ sources. The spectral indices are $\approx 1$. The coming into operation of the Owens Valley Radio Observatory (OVRO) two movable element interferometer at $960 \mathrm{MHz}$ (4 E-W and $4 \mathrm{~N}-\mathrm{S}$ regularly spaced baselines, up to1557 $\lambda$, which give a $\lesssim 10$ " positional accuracy) allows a new step forward.

After completion of the first of the two 90-ft paraboloids, Harris \& Roberts (1960) start a program to measure the flux density of 106 radio sources (CTA survey), mostly from the $3 C$ survey and compute the two-frequency spectral index $\alpha$ for 80 of them. They find $0.3<$ $\alpha<0.9$ for $80 \%$ of the objects, with median $\alpha \approx 0.6$. The narrow range of values for $\alpha$ implies that in all sources relativistic electrons have a very similar spectral energy distribution.

Moreover they find a few low frequency inverted spectra (e.g. CTA 21 and CTA 102, missing, for this reason, from the $3 C$ catalogue). This will be explained as due to Synchrotron SelfAbsorption (SSA) in sub-arcsec structures, impossible to observe with present epoch radio telescopes (Slish. 1963, Williams, 1963; see also Le Roux, 1961)

Conway et al. (1963) (CKL) coordinate a project Jodrell Bank-Cambridge-Caltech and provide a 9-v spectral analysis, in the interval $38<v<3200 \mathrm{MHz}$; for 160 radio sources (mostly $3 C R$ ). They find that in 134 radio sources the spectrum, at $v<1400 \mathrm{MHz}$, is a power-law, with $0.61<\alpha<0.81$ (average 0.71). In the remaining non-galactic objects high- $v$ steepening and low- $v$ flattening may occur. In 1964-1966 Kellermann will develop the complete analysis.

- High resolution and accurate positions for optical ID

The pressing request for very accurate radio positions, necessary to obtain safe optical $I D$, begins to be satisfied thanks to the two element OVRO interferometer.

Meanwhile, in 1958 the Palomar Sky Survey (PSS), with the 48" Schmidt telescope (begun in 1948) has been completed. Using the PSS two-color plates, hundreds of radio sources are more easily identified, with an obvious huge impact on the understanding of their nature.

In the second half of the $60 \mathrm{~s}$, the accurate positions by the Parkes radio telescope will allow to obtain a large amount of optical $I D$ in the Southern hemisphere.

The boom of optical ID reveals mostly radio galaxies, several of which optically quite faint. Radio galaxies will be used as standard candles for cosmological tests (Sandage, 1972), therefore with an interesting impact also on this field.

The extensive use of PSS also brings, approximately in 1961, to the ID of a few radio sources with star-like blue objects. For some time astronomers wonder whether radio stars, abandoned in late 1960, have been, after all, discovered. Optical spectra, however, are hard to interpret unless one applies large redshifts which put the re-born radio stars at cosmological distances. Obviously we are talking of the Quasars. The story of this discovery is very complex and we skip it here, but interested people may enjoy reading the paper by Kellermann (2013) who also reveals "spicy" background and gossips, not evident from the published papers.

\section{- $\quad$ High resolution and accurate morphology}

Large improvements in the determination of the radio source morphology are obtained by Maltby \& Moffet (1962): they observe with the OVRO interferometer (resolution $\approx 30$ ") 195 radio sources, mostly from the CTA survey (Harris \& Roberts, 1960), $90 \%$ of which are extragalactic. Using Fourier Inversion and/or model-fitting of the nine (including zero-baseline) complex visibility points they determine the (two-dimensional) structure of the radio sources. Almost half have two components which, when an associated optical object exists, are on either side of it. $\operatorname{Cyg} A$ is the prototype.

Great impact on the physics of radio sources!

- Lequeux (1962) observes, with the Nançay two movable antenna interferometer at $1420 \mathrm{MHz}$ (baselines: $6950 \lambda$ in EW, $1820 \lambda$ in NS; resolution $\approx 10$ ") about 40 powerful radio sources, 24 of which extragalactic. He confirms the double structure for $>50 \%$ of them. 
- Allen et al. (1962) discuss 133 out of the $3843 C(R)$ and $M S H$ radio sources, observed at 159 $\mathrm{MHz}$ with the long baseline, microwave-linked, EW interferometer (Jodrell Bank $250 \mathrm{ft}+$ 4 remote stations, 4 baselines from $2200 \lambda$ to $61100 \lambda$, resolution $\approx 1$ "). They find double or more complex structures for the large majority of the objects, with components 1-100" in size (average 30") and separated 10-600". These data will become soon very useful to search for far away radio sources, assumed to be those with smaller angular diameters.

\section{The 60s: the epoch of the Northern Cross}

Many problems (extragalactic nature of radio stars, synchrotron mechanism, ....) have been solved, technology has improved, money is (sometimes) coming. In these years one sees the flowering of a variety of radio telescopes. We mention some:

Aperture Synthesis: (Cambridge): 4C interferometer (1.2 km EW ×1 km NS); One-Mile interferometer (Earth Rotation Synthesis)

Large dishes:Jodrell Bank Mk I, (250-ft); Parkes, (210-ft); Green Bank, (300-ft, 140-ft)

Cross Radio Telescopes: Molonglo Cross: (1.6 km × 1.6 km); DKR-1000 (Pushchino,

$1 \mathrm{~km} \times 1 \mathrm{~km}) ;$ multi- $\lambda(2.5-10 \mathrm{~m}) ; \quad$ Northern Cross $(600 \mathrm{~m} \times 600 \mathrm{~m})$

Many deep, large area surveys are produced, at frequencies up to $5 \mathrm{GHz}$, counting up to a few thousands of radio sources.

It is in this rich exciting atmosphere that Italy joins radio astronomy with the construction of the Northern Cross radio telescope. To celebrate its $51^{\text {st }}$ anniversary, occurring exactly in this week, we spend now some words on its story. Then we terminate our tale.

In 1959 the director of the Physics Institute in Bologna (prof. G, Puppi, elementary particles physicist) comprehending the importance of radio astronomy as a new branch of physics and cosmology, succeeds in getting some funding from Ministry of Public Education (800 ML Lire $\approx 400,000 €$ ) to start radio astronomical research in Italy. He involves, as project manager, another elementary particle physicist, M. Ceccarelli (32 years old). More people enter into the project: G. Righini, G. Mannino (astronomers, who will give up), G. Sinigaglia (electronic engineer) and two students (A. Braccesi and G. Setti). Nobody has experience in the field....., so contacts are being taken for advice with Mills, Cambridge group, Christiansen and Högbom.

In order to practice, they build in a farmer yard at Medicina (Bologna), a prototype parabolic cylinder (familiarly called "Medicinoscope") $110 \mathrm{~m} \times 7 \mathrm{~m}$, operating a $327 \mathrm{MHz}$ (Braccesi et al., 1960) and a map of the galactic plane is produced (Braccesi

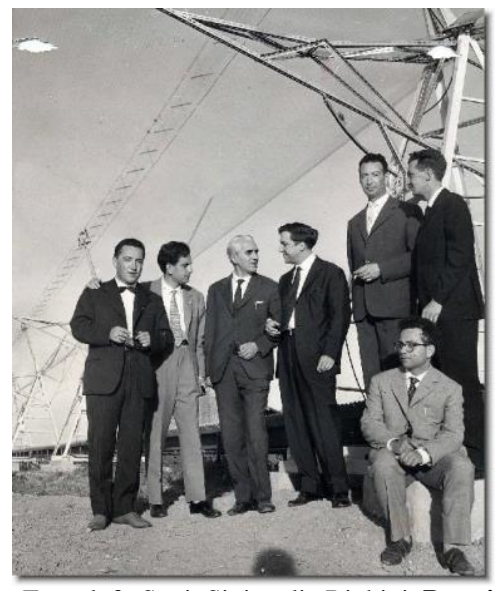

From left: Setti, Sinigaglia,Righini, Puppi, Mannino,_Ceccarelli, Braccesi (sitting) \& Vespignani, 1964). The good operation of the Medicinoscope allows to start the big project.

The design is for a Mills Cross type telescope of $\approx 1.2 \mathrm{~km} \times 1.2 \mathrm{~km}$, operating at $408 \mathrm{MHz}$, but it is soon realized that a $\boldsymbol{T}$ of $\approx 0.6 \mathrm{~km} \times 1.2 \mathrm{~km}$ in size is equivalent. The ambitious purpose is to carry out a large ( $>10^{4}$ sources) deep (tenths of Jy) survey in order to build an accurate confusion-free $\log N-\log S$ which may set a final word on the $S S T$ debate, still alive in this period. With the recruitment of D. Harris and L. Volders (astronomers), G. Gelato and C. Rosatelli (electronic engineers), several technicians (and, a bit later, the two of us, undergraduate) the ROUB (Radio Osservatorio Università Bologna) group is formed.

In 1964, because of delays in arrival of the last tranche of funding, only EW arm $(564 \mathrm{~m} \times 35 \mathrm{~m})$ and a chunk $(320 \mathrm{~m} \times 47 \mathrm{~m})$ of the NS arm are up. It is decided to put at least the EW arm into operation, hoping in a better future. The EW arm is electrically divided into 6 sections which are then combined to get three independent simultaneous EW fan beams (HPBW 4’x110').

The Cross opening is on Oct. $24^{\text {th }}, 1964$. 
Observations are conducted at Christmas time, at night (free of man-made interferences), i..e. $0 \mathrm{~h}<R A<13 \mathrm{~h}$, in the sky strip $\left(-30^{\circ}<\right.$ dec $\left.<-20^{\circ}\right)$ overlapping the Parkes survey at $400 \mathrm{MHz}$. The noise is $\approx 0.06 \mathrm{Jy}$ but only sources with $S>1 \mathrm{Jy}$ are extracted, to reduce confusion problems. The B1 Survey is quickly compiled from the hand-made average of the chart records of the three EW beams. It counts 624 radio sources in 0.6 ster (Braccesi et al, 1965).

By the end of ' 66 , the ongoing financial problems let foresee an uncertain future for the completion of the Cross, therefore, with the last money at disposal, it is decided to put the incomplete NS arm into operation.

At Christmas time (again....) 1967, the $600 \mathrm{~m} \times 300 \mathrm{~m}$ telescope is ready. Three NS beams are multiplied with those in EW, giving $3 \times 3\left(5 \times 3\right.$ after 1970) simultaneous pencil beams $\left(3^{\prime} \times 10^{\prime}\right)$. Grueff \& Vigotti (1968) realize a test Mini-survey $(G V)$ in the sky area $07 \mathrm{~h} 40 \mathrm{~m}<R A<18 \mathrm{~h} 20 \mathrm{~m}$, $34^{0}<d e c<35^{\circ}$. It counts 328 radio sources with $S>0.15 \mathrm{Jy}$ in 0.04 ster. (confusion at $\approx 0.25 \mathrm{Jy}$ ). Between autumn ' 68 and end ' 72 the $B 2$ survey ( 4 sections, references in Fanti et al., 1974), in the strip $21^{\circ} 40^{\prime}<$ dec $<40^{\circ} 18^{\prime}$ is completed. It counts $\approx 10^{4}$ radio sources down to $S_{\text {min }}=0.2$ Jy.

In the period 1972-76 the entire electronics get improved and the NS arm extended to $600 \mathrm{~m}$

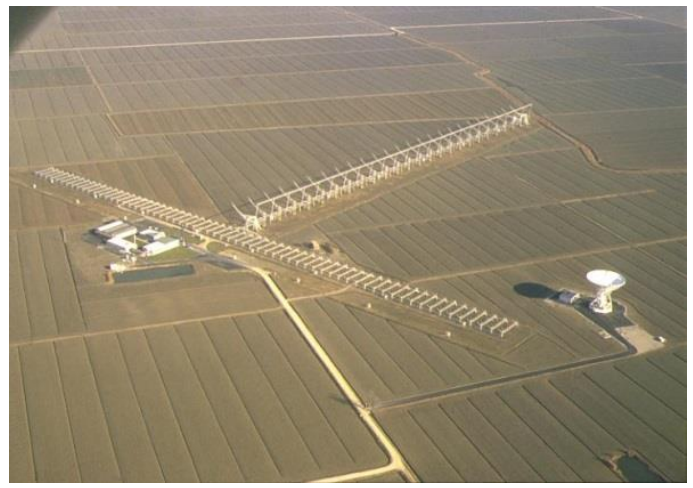
by cutting the existing $320 \mathrm{~m}$ NS arm and redistributing the structure in length, so as to obtain an array twice as long and a half as wide (financed now by the CNR). The beam, at zenith, is $2.6^{\prime} \times 4.8^{\prime}$. The novel NS arm is divided into 8 sections and the final Cross works as a Synthesis Telescope with $6 \times 8$ simultaneous complex correlations. A $0.5^{\circ}$ sky strip can be synthetized in each scan. The B3 survey (Ficarra et al., 1985) covers $35^{\circ} 45^{\prime}<$ dec $<49^{\circ} 15^{\prime}$ and counts 13354 radio sources down to $S \sim 0.1 \mathrm{Jy}$.

The Northern Cross provided the scientific community with a large set of data, extensively used by many people to study, at a frequency different from that of the $3 C R$ and at much lower flux densities, extended samples of extragalactic radio sources.

The Bologna surveys allowed to bridge the gap in luminosity between the high power $3 C R$ and the 10-mJy surveys (5C at this epoch; see e.g. Wall \& Benn, 1982).

After all the original "ambitious purpose" has been achieved....

The onset of a solid research group led to the creation in 1970 of IRA (Istituto di RadioAstronomia) of CNR (National Research Council) with permanent scientific and technical staffs and regular funding. The $C N R$ financed the construction of the two $32 \mathrm{~m}$ VLBI parabolae (Medicina \& Noto) and supported for years the VLBI operations in Italy. It has also promoted the $64 \mathrm{~m}$ SRT project in Sardegna. All three antennas were designed and managed by IRA.

\section{References}

H. Alfvén, 1937, Ark, Mat. Astr. Fys, 25B, No. 29

H. Alfvén, N. Herlofson, Cosmic Radiation and Radio Stars 1950, Phys. Rev., 78, 616

L.R. Allen, R. H-Brown, H,P, Palmer An analysis of the angular sizes of radio sources, 1962 , MNRAS 125, 57

W. Baade, Polarization in the jet of Messier 87, 1956, ApJ 123, 350

W. Baade, R. Minkowski Identification of the Radio Sources in Cassiopeia, Cygnus A and Puppis A, 1954a, ApJ 119, 206

W. Baade, R. Minkowski On the Identification of Radio Sources 1954b, ApJ 119, 215

A.S. Bennett, The preparation of the revised $3 C$ catalogue of radio sources, 1962, MmRAS 68, 63

B. Bertotti, R.Balbinot, S. Bergia, A.Messina Modern Cosmology in Retrospect. 1991, Cam.Univ.press

J.G. Bolton, Discrete sources of galactic radio frequency noise, 1948, Nature, 162, 141

J.G. Bolton, G.J., Stanley, Variable source of radio frequency radiation in the constellation of Cygnus, 1948, Nature, 161, 312

J.G. Bolton, G.J. Stanley, The position and probable identification of galactic radio-frequency radiation Taurus-A" 1949, AuSRA, 2,139 
J.G, Bolton, G.J. Stanley, O.B. Slee, Positions of three discrete sources of galactic radio frequency radiation, 1949, Nature, 164, 102

J.G. Bolton, K.C. Westfold, Galactic radiation at radio frequencies. IV: The distribution of radio star in the galaxy, 1951, AuSRA 4, 476

J.G. Bolton, G.J. Stanley, O.B. Slee, Galactic radiation at radio frequencies, 1954, AuJPh 7, 11

J.G. Bolton, Distribution of radio stars, 1956, Obs. 76, 621

H. Bondi, T. Gold, The Stady-State Theory of the Expanding Universe 1948, MNRAS 108, 252

A. Braccesi, M.Ceccarelli, G. Mannino, et al. Operation at the Medicina Station of a $327 \mathrm{MHz}$ Radiotelescope, 1960, Nuovo C. X 17, 614

A, Braccesi, G. Vespignani, A map of the $327 \mathrm{MHz}$ continuoum radio emission of the sky between $\alpha=16 h$ and $\alpha=24 h$ from $\delta=-20^{\circ}$ to $\delta=+60^{\circ}, 1964$, Nuovo C. 31(2), 310

A. Braccesi, M. Ceccarelli, R. Fanti et al. A catalogue of $<$ radiosources from $\delta=-30^{\circ}$ to $\delta=-20^{\circ}$ at $408 \mathrm{MHz}, 1965$, Nuovo C. X 40, 267

G.R. Burbidge, On Synchrotron radiation from Messier 87, 1956, ApJ 124, 416

G.R. Burbidge, Estimates of the Total Energy in Particles and Magnetic Field in the Non-Thermal Radio Sources, 1959, ApJ 129, 849

M.H. Cohen, The Owens Valley Radio Observatory: the Early Years, 1994, Caltech Library

R.G. Conway, K.I. Kellermann, R.J. Long, The radio frequency spectra of discrete radio sources, 1963 MNRAS 125, 261

V.A. Dombrovsky, On the nature of the radiation of the Crab nebula, 1954, Doklady Akad. Nauk USSR 94,1021

J.A. Earl Cloud-Chamber Observations of Primary Cosmic-Ray Electrons, PhRvL, 6, 125

D.O. Edge, P.A.G. Scheuer, J.R. Shakeshaft, Evidence on the spatial distribution of radio sources derived from a survey at a frequency of $159 \mathrm{Mc} / \mathrm{s}, 1958$, MNRAS 118, 183

C. Fanti, R.Fanti, A. Ficarra. L. Padrielli, The B2 catalogue of radio sources - fourth part, 1974, A\&AS 18,147

A. Ficarra, G. Grueff, G. Tomassetti, A new Bologna sky survey at 408 MHz, 1985, A\&AS 59, 55

H. T. Friis \& C.B. Feldman, A multiple Unit Steerable Antenna for Short-Wave Reception 1937 , Proc. IRE, 25, 841

V.L. Ginzburg, 1953, USP Phiz Nauk 51, 543 (in Russian)

J.F.R. Gower, The source counts from the 4C survey, 1966, MNRAS 133, 151

J.L. Greenstein, L.G. Henyey, P.C. Keenan, Interstellar Oriugin of Cosmic Radiation at Radio-Frequencies, 1946, Nature 157, 805

G. Grueff, M. Vigotti, A Pencil-Bem Deep Sky Survey at 408 MHz, 1968, ApL 1, 113

J.S. Hall, A.H. Mikesell Observations of polarized light from stars 1949, AJ, 54, 187

R. Hanbury Brown, A Symposium on Radio Astronomy, 1953, Obs 73,185

R. Hanbury Brown, C. Hazard, A survey of 23 localized radio sources in the northern Emisphere, 1953 MNRAS 113, 123

D.E. Harris, J.A. Roberts Radio source measurements at 960 Mc/s, 1960, PASP 72, 237

L.G. Henyey, P.C. Keenan, The interstellar Radiation from Free Electrons and Hydrogen Atoms, 1940, ApJ 91, 625

J.S. Hey, Solar Radiations in the 4-6 Meter Radio Wave-Length Band 1946a, Nature 157, 47

J.S. Hey, S.J. Parsons, J.W. Phillips, Fluctuations in cosmic radiation at radio-frequencies, 1946b, Nature 158, 234

W.A. Hiltner, Polarization of Radiation from Distant Stars by the Interstellar Medium 1949, Nature 163,283

F. Hoyle, A new model for the Expanding Universe 1948, MNRAS 108, 372

E. Hubble, M.L. Humason,The velocity-distance relation among extragalactic nebulae, 1931, CMWCL 427, 1

K.I. Kellermann, The discovery of quasars and its aftermath 2013, Journal of Astronomical History and Heritage, 17, 267

K.O. Kiepenheur, Cosmic rays as the source of general galactic radio emission, 1950, Ph.Rev. 79,738

K.G. Jansky, Electrical Disturbances apparently of extraterrestrial origin, 1933, Proc.IRE. 21, 1387

C.H. Mayer, T.P. McCullough, R.M., Sloaneker, Evidence for polarized radio radiation from the Crab Nebula, 1957, ApJ 126, 468

J. Lequeux, Mesures interférométrique à haute resolution et de l structure des principals radio sources à $1420 \mathrm{MHz}, 1962$, AnAp 25,221

E. Le Roux Etude théorique du rayonnement synchrotron des radiosources, 1961, AnAp 24, 71 
P. Maltby, A.T. Moffet, Brightness distribution in discrete radio sources. III The structure of the sources 1962, ApJS 67, 93

C.H. Mayer, T.P. McCullough, R.M., Sloaneker, Polarization of the Radio Emission of Taurus A, Cygnus A and Centaurus A, 1962, AJ 67, 581

B.Y. Mills, The distribution of discrete sources of cosmic radiation 1952a, AuSRA 5, 266

B.Y Mills, The position of six discrete sources of cosmic radiation, 1952b, AuSRA 5, 456

B.Y. Mills, O.B. Slee, A preliminary survey of radio sources in a limited region of the sky at a wavelength of $3.5 \mathrm{~m}, 1957$, AuJPh, 10, 162

B.Y. Mills, The radio continuum radiation from the Galaxy, 1959a, PASP 71, 267

B.Y. Mills, Galactic structure a meter wavelengths, 1959b, IAUS n.9, 431

B.Y. Mills, O.B. Slee, E.R. Hill A survey of radio sources between declination $-50^{\circ}$ and $-80^{\circ} .1961$, AuPh 14, 497

L.A. Moxon, Variation of Cosmic Radiation with Frequency, 1946, Nature 158, 758

J.H.Oort, T. Walraven, Polarization and composition of the Crab nebula, 1956, BAN, 12285

J.L. Pawsey, Preliminary statistics of discrete sources obtained with the 'Mills Cross', 1957, IAUS 4, 228

G.W. Potapenko \& D.F. Folland, 1936, Science News Letter 131

V.A. Razin, The Polarization of Cosmic Radio Radiation at Wavelengths of 1.45 and 3.3 Meters, 1958, AZh 35, 241

G. Reber, Galactic radio waves, 1950, ASPL 259, 67

M. Ryle, F.G. Smith, A new intense source of radio frequency radiation in the constellation of Cassiopeia, 1948, Nature, 162, 462

M. Ryle, Radioastronomy, 1950, Report on Progress in Physics., vol. 13, p. 184

M. Ryle, A. Hewish, The effects of the terrestrial ionosphere on radio waves from discrete sources in the Galaxy 1950, MNRAS 110, 381

M. Ryle, F.G. Smith, B. Elsmore. A preliminary survey of the radio stars in the northern Hemisphere, 1950, MNRAS 110, 508

M. Ryle, Radio stars and their cosmological significance, 1955, OBS 75, 187

A.K. Sandage, The Redshift-Distance Relation. III. Photometry and the Hubble Diagram for Radio Sources and the Possible Turn-On Time for QSOS, 1972, AJ 178, 25

P.A.G. Scheuer, A statistical method for analyzing observations of faint radio stars, 1957, Math.Proc. Camb. Phil. Soc. 53, 764

P.F. Scott, M. Ryle, The number-flux density relation for radio sources away from the galactic plane, 1961, MNRAS 122, 389

J.R. Shakeshaft, M. Ryle, J.E. Baldwin et al. A survey of radio sources between declinations $-38^{\circ}$ and $+83^{\circ}, 1955$, MNRAS 67, 106

C.A. Shain, C.S. Higgins, Observations of the general background and discrete sources of $18.3 \mathrm{Mc} / \mathrm{s}$ cosmic noise, 1954. AuJPh, 7, 460

I.S. Shklovsky, 1953, Dokl. Akad Nauk SSSR 90, 983 (in Russian)

G.J. Stanley, O.E. Slee, Galactic radiation at radio frequencies. II the discrete sources, 1950, AuSRA 3, 234

V.I.Slish, Angular size of radio Stars, 1963, Nature 199, 682

J.M. Thomson, An Attempt to detect Linearly Polarized Radio Emission from the Galaxy, 1957, Nature 180,495

C.H. Townes, Interpretation of the Radio Radiation from the Milky way, 1947, ApJ 105, 235

A. Unsöld Uber den Ursprung der radiofreqyenz strahkung und der Ultrastrahlung in der Milchsrasse 1949, ZA, 26, 176

M. Vashakidze, On the degree of polarization of the radiation of near-by extra-galactic nebulae and of the Crab nebula”, 1954,, Astr. Circ. No 147

J.V. Wall, C.R.Benn Cosmological evolution of QSOs and radio galaxies from radio-selected samples 1982, IAUS n.97, 441

G.Westerhout., J.H. Oort, A comparison of the intensity distribution of radio-frequency radiation with a model of the galactic system 1951, BAN. 11, 323

$\mathrm{G}$. Westerhout, A suvey of the countinuous radiation from the galactic system at a frequency $1390 \mathrm{Mc} / \mathrm{s}$, 1958, BAN 14, 215

G. Westerhout, C.L. Seeger, W.N. Brouw et al. Polarization of the galactic 75-cm Radiation, 1962, BAN 16, 187

G.R. Whitfield, The spectra of radio stars, 1957, MNRAS 117, 680

P.I.S Williams, Absorption in Radio Sources of High Brightness Temperature”, 1963, Nature, 200, 56 